\title{
One-Dimensional Transient Response of the Inner Magnetosphere at the Magnetic Equator, 2. Analysis of Waveforms

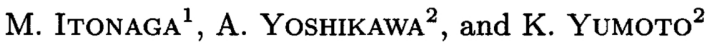 \\ ${ }^{1}$ Computation Center, Kyushu University, Fukuoka 812-81, Japan \\ ${ }^{2}$ Department of Earth and Planetary Sciences, Kyushu University, Fukuoka 812-81, Japan
}

(Received July 8, 1996; Revised November 1, 1996; Accepted November 12, 1996)

Under a model of altitude distribution of the Alfvén speed $V_{\mathrm{A}}$, one-dimensional transient response of the inner magnetosphere at the magnetic equator to earthward propagating impulse- and step-like MHD disturbances is considered. The waveforms of transient compressional oscillations due to these disturbances at some $L$ shells are directly simulated by a numerical inversion of the Laplace transform with orthonormal Laguerre functions. The present paper concentrates on the analysis of waveforms. Then, it is verified that the compressional oscillations are due to the poles of the system under consideration. The oscillation arising from the cavity resonance all over the inner magnetosphere is most dominant. However, its amplitude becomes smaller as the characteristic time scale $T$ of an incident disturbance grows large, and it is negligibly small for $T$ greater than several times of eigenperiod of the resonance. On the other hand, when $T$ is relatively small (e.g., $T \lesssim 10 \mathrm{~s}$ ), the oscillations due to the cavity resonances trapped around the trough in $V_{\mathrm{A}}$ are outstanding. It is also found that the relative phase between the cavity-mode oscillations all over the inner magnetosphere at the earth's surface and another $L$ shell increases monotonically with $L$ when the inner magnetosphere has no strong gradient or a strong positive gradient of $V_{\mathrm{A}}$ at its outer boundary. However, the relative phase is nearly zero and nearly $180^{\circ}$ inside and outside a specific $L$ shell, respectively, when the inner magnetosphere has a strong negative gradient at its outer boundary. The one-dimensional cavity-mode type resonance of the inner magnetosphere is certainly a cause of equatorial Pi2 pulsations. However, some constituents of the Pi2's may be not cavity-mode oscillations but quasi-steady-state oscillations forced by some damped sinusoidal waves incident on the outer boundary of the inner magnetosphere.

\section{Introduction}

Using magnetic field records acquired in the inner magnetosphere $(L=2-5)$ by the AMPTE CCE satellite, Takahashi et al. (1992) identified 25 equatorial Pi2 events whose waveforms match those simultaneously observed at Kakioka ground station located at $L=1.23$. All events except one were observed within 3 hours of midnight. They argued that Pi2 pulsations at $L \lesssim 5$ arise from a cavity-mode type resonance excited in the inner magnetosphere bounded at low and high altitudes by the ionosphere and an Alfvén velocity gradient, respectively. They also claimed that the cavity resonance is probably excited by earthward propagating MHD compressional waves launched at the substorm onset by a large scale magnetic reconfiguration associated with the cross-tail current disruption. In order to explain the longitudinal localization of the compressional oscillation in the inner magnetosphere Takahashi et al. (1995) regarded equatorial Pi2's as something analogous to sound waves emitted from a point source between two walls with a finite cross section for reflection (see Fig. 18 in their paper). The point source corresponds to the region of current disruption. If the walls are parallel to each other, long-lived echoes (or Pi2's) will be observed only near the source longitude, because the waves that are obliquely incident on the walls will be lost by the longitudinal propagation out of the system. Thus, it appears that one-dimensional response of the inner magnetosphere (or plasmasphere) along the source 
longitude around the magnetic equator plays a significant role in the compressional oscillation responsible for the Pi2's at $L \lesssim 5$.

In the present study, using a model of altitude distribution of the Alfvén speed $V_{\mathrm{A}}$, we will consider one-dimensional transient response of the inner magnetosphere at the magnetic equator to earthward propagating impulse- and step-like MHD disturbances. We will directly simulate waveforms of transient compressional oscillations due to these disturbances at some $L$ shells by a numerical inversion of the Laplace transform with orthonormal Laguerre functions, which has been developed by Weeks (1966), Lyness and Giunta (1986), and Garbow et al. (1988). Through in detail examining these simulated waveforms in connection with the observational results obtained by Kyushu University as well as those by Takahashi et al. $(1992,1995)$, then, we will tackle the problem whether the cavity-mode type resonance is the sole cause of the equatorial Pi2's.

Itonaga et al. (1996), which is hereafter referred to as paper 1, have investigated the transfer function of the system under consideration and its poles, and have provided foundations for discussions of the simulated waveforms. In paper 1 the following results are obtained. The poles, which are mathematical counterparts of the cavity resonances, appear owing to the inhomogeneity of $V_{\mathrm{A}}$ and their locations depend on the altitude distribution of $V_{\mathrm{A}}$ as well as the position of external source (or outer boundary of the inner magnetosphere). Even if there exists no strong Alfvén velocity gradient at the outer boundary, an observable cavity-mode oscillation in the Pi2 range can be excited because of the existence of the plasmapause. However, the existence of a strong Alfvén velocity gradient at the outer boundary brings about a long-lived nature of the cavity-mode oscillation as well as calls some new poles into existence. The surface of the solid earth forms the inner boundary at which the almost perfect reflection of wave takes place, while the ionosphere is only of secondary importance as a reflector of wave. The existence of the solid earth plays an essential role in the observability of the compressional oscillation arising from the cavity resonance all over the inner magnetosphere. The real part of each pole has a negative value, meaning that the cavity-mode oscillation decays with a damping factor of absolute value of the real part of the pole. Such a damping is primarily due to the leakage of energy through the outer boundary of the inner magnetosphere.

The present paper, which is a companion of paper 1, concentrates on the analysis of waveforms and gives an answer to the problem whether the cavity-mode type resonance is the sole cause of the equatorial Pi2's. Also in the present paper the model and notations in paper 1 as well as $\sigma_{\mathrm{E}}=0.001 \mathrm{~S} / \mathrm{m}$ and $\Sigma_{\mathrm{C}}=1 \mathrm{~S}$ are used, and model 1 designated by $\mathrm{AS}_{1}$ is employed as an altitude distribution of $V_{\mathrm{A}}$ (cf. Fig. 1 of paper 1).

\section{Numerical Inversion of the Laplace Transform}

Prior to the analysis of waveforms, we will give an outline of the numerical inversion of the Laplace transform used in the present study. For a given function $\varphi(\tau)$, it can be shown that there exists an expansion of $\varphi(\tau)$ in terms of orthonormal Laguerre functions $\Phi_{k}(\tau)$ 's (Weeks, 1966; Lyness and Giunta, 1986):

$$
\varphi(\tau)=\mathrm{e}^{\sigma \tau} \sum_{k=0}^{\infty} a_{k} \Phi_{k}(b \tau)
$$

for any parameters $b$ and $\sigma$ such that $b>0$ and $\sigma>\sigma_{0}$ with the convergence abscissa $\sigma_{0}$ of the Laplace transform $\hat{\varphi}(p)$ of $\varphi(\tau)$. Here, $\Phi_{k}(\tau)$ 's are given by the recurrence relation

$$
k \Phi_{k}(\tau)+(\tau-2 k+1) \Phi_{k-1}(\tau)+(k-1) \Phi_{k-2}(\tau)=0 \quad(k \geq 2)
$$

with

$$
\Phi_{0}(\tau)=\mathrm{e}^{-\tau / 2}, \quad \Phi_{1}(\tau)=(1-\tau) \Phi_{0}(\tau)
$$


The expansion coefficients $a_{k}$ 's coincide with the Taylor expansion ones of $\phi(z)$ defined by

$$
\phi(z)=\frac{b}{1-z} \hat{\varphi}\left(\frac{b}{1-z}-\frac{b}{2}+\sigma\right),
$$

which is regular within a circle $|z|<R_{\mathrm{c}}$ with $R_{\mathrm{c}} \geq 1$, and so they are given by

$$
a_{k}=\frac{r^{-k}}{2 \pi} \int_{0}^{2 \pi} \phi\left(r \mathrm{e}^{i \theta}\right) \mathrm{e}^{-i k \theta} d \theta
$$

with any parameter $r$ such that $0<r<R_{\mathrm{c}}$.

Given the function $\widehat{\varphi}(p)$, the problem is to construct an approximation

$$
\widetilde{\varphi}_{N}(\tau)=\mathrm{e}^{\sigma \tau} \sum_{k=0}^{N} \widetilde{a}_{k} \Phi_{k}(b \tau)
$$

to $\varphi(\tau)$, where $N$ is an integer and $\widetilde{a}_{k}$ 's are estimates of the expansion coefficients $a_{k}$ 's. The method for obtaining the estimates $\tilde{a}_{k}$ 's was first presented by Weeks (1966), which is called the Weeks method. More recently, Lyness and Giunta (1986) proposed a modification of the Weeks method. Our numerical inversion of the Laplace transform is performed using this modification. In the modified method, $\tilde{a}_{k}$ 's are given by

$$
\widetilde{a}_{k}=\frac{r^{-k}}{M} \sum_{l=0}^{M-1} \phi\left(r \mathrm{e}^{i 2 \pi l / M}\right) \mathrm{e}^{-i 2 \pi k l / M}
$$

with $N=M-1$. Note that the FFT method can be utilized when a power of 2 is employed as $M$. Further, if $\varphi(\tau)$ is a real-valued function, the computation of $\phi\left(r \mathrm{e}^{i 2 \pi l / M}\right)$ is required only for $l=0,1, \ldots, M / 2$ because the relation

$$
\phi\left(r \mathrm{e}^{i 2 \pi(M-l) / M}\right)=\phi\left(r \mathrm{e}^{i 2 \pi l / M}\right)^{*}
$$

holds for any integer $l$, where the superscript $*$ denotes the complex conjugate. As for the selection of $b, \sigma$ and $r$ for a given integer $M$ and a given upper limit $\tau_{\max }$ such that the approximation of $\varphi(\tau)$ holds in the range of $\tau=0$ to $\tau=\tau_{\max }$, refer to Garbow et al. (1988).

\section{Analysis of Waveforms}

\subsection{Step-like disturbances}

In this subsection we will consider the transient response of the inner equatorial magnetosphere to earthward propagating step-like MHD disturbances. We will simulate waveforms of compressional oscillations due to these disturbances at some $L$ shells by the numerical inversion of the Laplace transform with orthonormal Laguerre functions outlined in the last section. The step-like disturbance incident on the outer boundary of the inner magnetosphere, which begins at $\tau=0$, is represented by

$$
\delta B_{\mathrm{in}}(\tau)= \begin{cases}\frac{\delta B_{\max }}{2}[1-\cos (\pi \tau)] & (0 \leq \tau<1) \\ \delta B_{\max } & (\tau \geq 1)\end{cases}
$$

with a maximum amplitude $\delta B_{\max }$. Then, we have

$$
\delta \widehat{B}_{\text {in }}(p)=\frac{\delta B_{\max }}{2} \frac{\pi^{2}}{p\left(p^{2}+\pi^{2}\right)}\left(1+\mathrm{e}^{-p}\right)
$$


by Laplace-transforming $\delta B_{\text {in }}(\tau)$. Note that the characteristic time scale $T$ is a rise time of the disturbance. These disturbances are something analogous to bay- or SC-type disturbances.

Figure 1 shows transient waveforms (solid lines) of the compressional component $\delta B_{x}$ of the perturbation magnetic field, which are normalized by $\delta B_{\max }\left(\delta B_{\max }=1\right)$, simulated at the earth's surface $(L=1)$ for the incident step-like disturbances (dashed lines) at $L=7$ with $T=10,30$, $60,120,300$ and $600 \mathrm{~s}$. Here, the inner magnetosphere has no strong Alfvén velocity gradient at its outer boundary $\left(V_{n}=\mathrm{AS}_{1}\left(L_{n}\right)\right)$. The travel time of wave across the $L$ range of 1 to 7 , which is estimated from model 1 for the altitude distribution of $V_{\mathrm{A}}$ (see Fig. 1 in paper 1), is $34.4 \mathrm{~s}$ and is indicated by the arrows in the figure. It is found in Fig. 1 that some oscillations are excited. As illustrated later, these oscillations originate in the poles of the system under consideration. While $L_{n}=7(n=599)$ in Fig. 1, the similar waveforms are also obtained for other values of $L_{n}$ so far as $L_{n}$ is greater than 5 , although the amplitude of oscillation decreases as $L_{n}$ increases. In general, as seen from the figure, the oscillation arising from the cavity resonance all over the inner magnetosphere is most predominant. However, its amplitude becomes smaller as $T$ grows large, and it is negligibly small for $T$ greater than several times of eigenperiod of the resonance. On the other hand, when $T$ is relatively small (e.g., $T \lesssim 10 \mathrm{~s}$ ), the oscillations due to the cavity resonances trapped around the trough in $V_{\mathrm{A}}$ are outstanding. We have found that these characteristics are independent of whether or not the inner magnetosphere has a strong Alfvén velocity gradient at its outer boundary (not shown).

Figure 2 presents a comparison of the waveform (solid lines) at $L=1$ with those (dashed lines) at $L=2,3,4,5,6$ and 7 in case of $L_{n}=7$ and $T=60 \mathrm{~s}$. The similar waveforms are found in the inner magnetosphere. The oscillation originating in the cavity resonance all over the inner magnetosphere is also most predominant at any $L$ shell. Then, it might be expected that the relative phase between the oscillations at the earth's surface and another $L$ shell is near zero and near $180^{\circ}$ at $L<4.15$ and $L>4.15$, respectively, according to the phase structure in the case (a) of Fig. 4 in paper 1. Such a structure is also reported in Takahashi et al. $(1992,1995)$. However, that is not the case and it appears that the relative phase increases monotonically with $L$ (Fig. 3 ). Figure 3 displays a superposition of the waveforms at $L=1,2,3,4,5,6$ and 7 in the ordinate range of 1.7 to 2.7. It should be noted that the phase structures in Fig. 4 of paper 1 are those of the steady-state oscillations forced by monochromatic waves incident on the outer boundary of the inner magnetosphere. The phase structure similar to that in the case (a) of Fig. 4 in paper 1 will be of course realized in the resonance between two rigid walls at which the perfect reflection of wave takes place. In the system under consideration, however, although the wave is almost perfectly reflected at the inner boundary (or earth's surface), there exists the leakage of energy, which is responsible for the damping of oscillation, through the outer boundary. Then, since the oscillations found in Fig. 2 are not steady-state oscillations forced by monochromatic waves, the relative phase has a quite different structure.

The compressional component $\delta B_{x}$ undulates around the eventual level 2 at any $L$ shell (Fig. 2). Then, considering $\delta B_{x}^{\prime}=\delta B_{x}-2$, we sample $\delta B_{x}^{\prime}$ with an interval of $\Delta \tau=0.02$, corresponding to $1.2 \mathrm{~s}$ for $T=60 \mathrm{~s}$, from the instant when $\delta B_{x}^{\prime}$ first reaches the zero level and denote the samples by $s_{n}(n=0,1, \ldots)$. Now, to demonstrate that the oscillations found in Fig. 2 arise from the poles of the system under consideration, assuming a model

$$
s_{n}=\sum_{k=1}^{m} A_{k} \mathrm{e}^{-\alpha_{k} n \Delta \tau} \cos \left(\omega_{k} n \Delta \tau+\theta_{k}\right) \quad(n=0,1, \ldots, N-1),
$$

we estimate the parameters (damping factor $\alpha_{k}$, angular frequency $\omega_{k}$, initial amplitude $A_{k}$, and initial phase $\theta_{k}$ ) using a modified Pisarenko method (Itonaga, 1990). The number $m$ of exponentially damped sinusoids is determined using the minimum description length (Rissanen, 1978). In the following $N=200$ is employed as the number of samples. When the estimates 

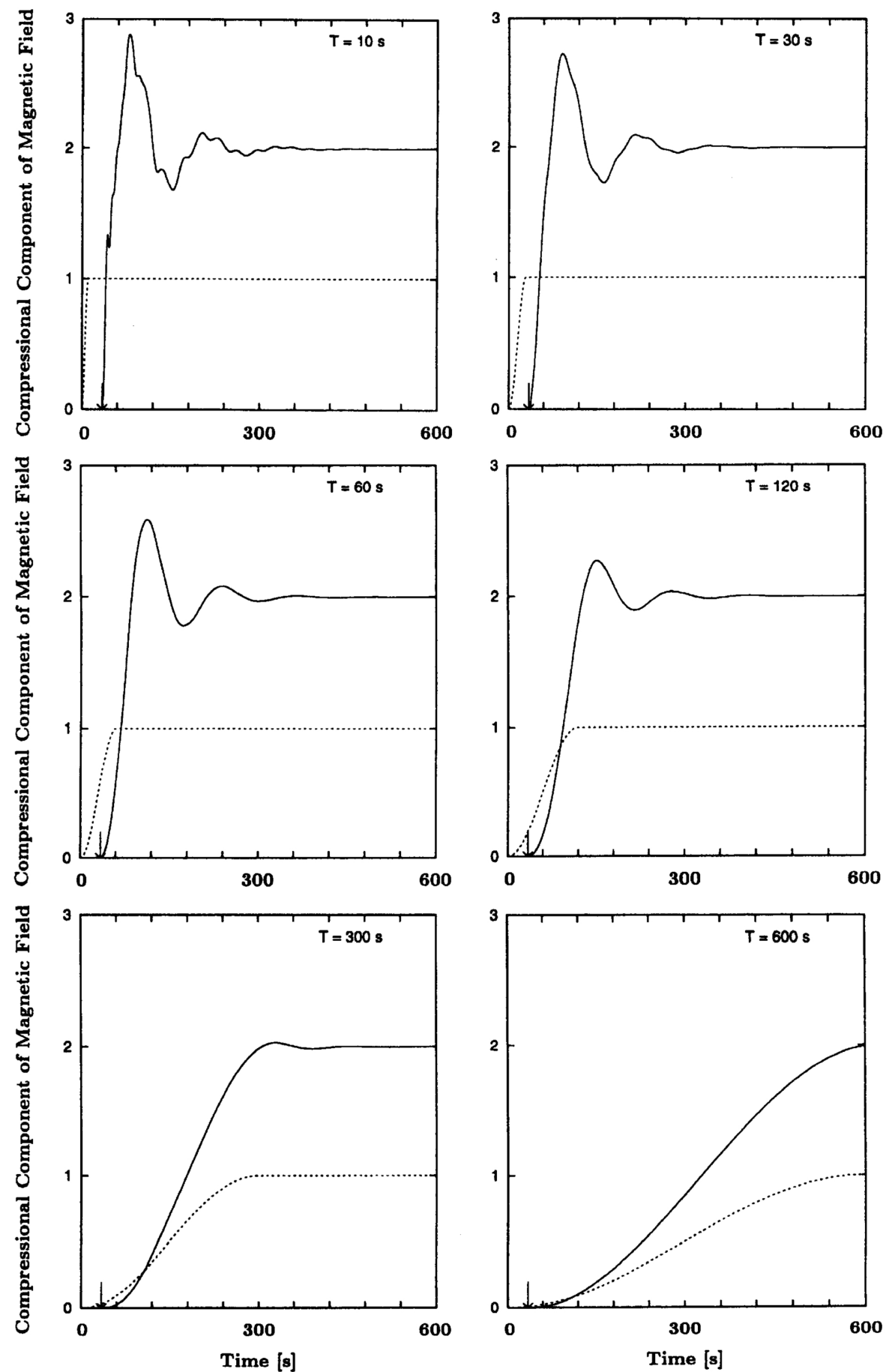

Fig. 1. Transient waveforms (solid lines) of the compressional component $\delta B_{x}$ simulated at $L=1$ for incident step-like disturbances (dashed lines) at $L=7$ with $T=10,30,60,120,300$ and $600 \mathrm{~s}$ when the inner magnetosphere has no strong Alfvén velocity gradient at its outer boundary $\left(V_{n}=\operatorname{AS}_{1}\left(L_{n}\right)\right)$. Here, model 1 $\left(\mathrm{AS}_{1}\right)$ is employed for the altitude distribution of $V_{\mathrm{A}}$, and the values of $\sigma_{\mathrm{E}}=0.001 \mathrm{~S} / \mathrm{m}$ and $\Sigma_{\mathrm{C}}=1 \mathrm{~S}$ are used. 

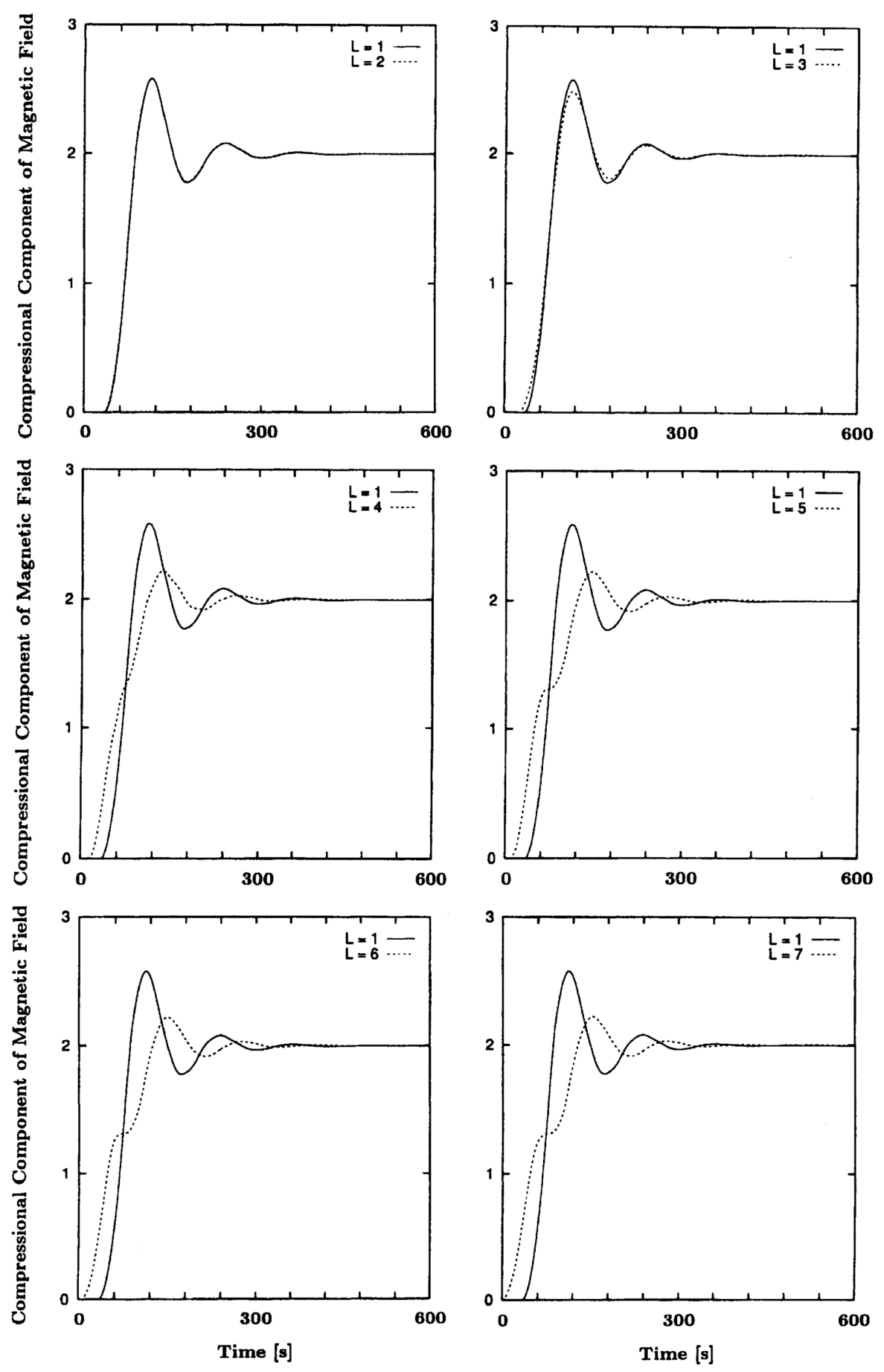

Fig. 2. Comparison of the waveform (solid lines) at $L=1$ with those (dashed lines) at $L=2,3,4,5,6$ and 7 for the incident step-like disturbance at $L=7$ with $T=60 \mathrm{~s}$ when the inner magnetosphere has no strong Alfvén velocity gradient at its outer boundary $\left(V_{n}=\operatorname{AS}_{1}\left(L_{n}\right)\right)$. Here, model $1\left(\mathrm{AS}_{1}\right)$ is employed for the altitude distribution of $V_{\mathrm{A}}$, and the values of $\sigma_{\mathrm{E}}=0.001 \mathrm{~S} / \mathrm{m}$ and $\Sigma_{\mathrm{C}}=1 \mathrm{~S}$ are used. 


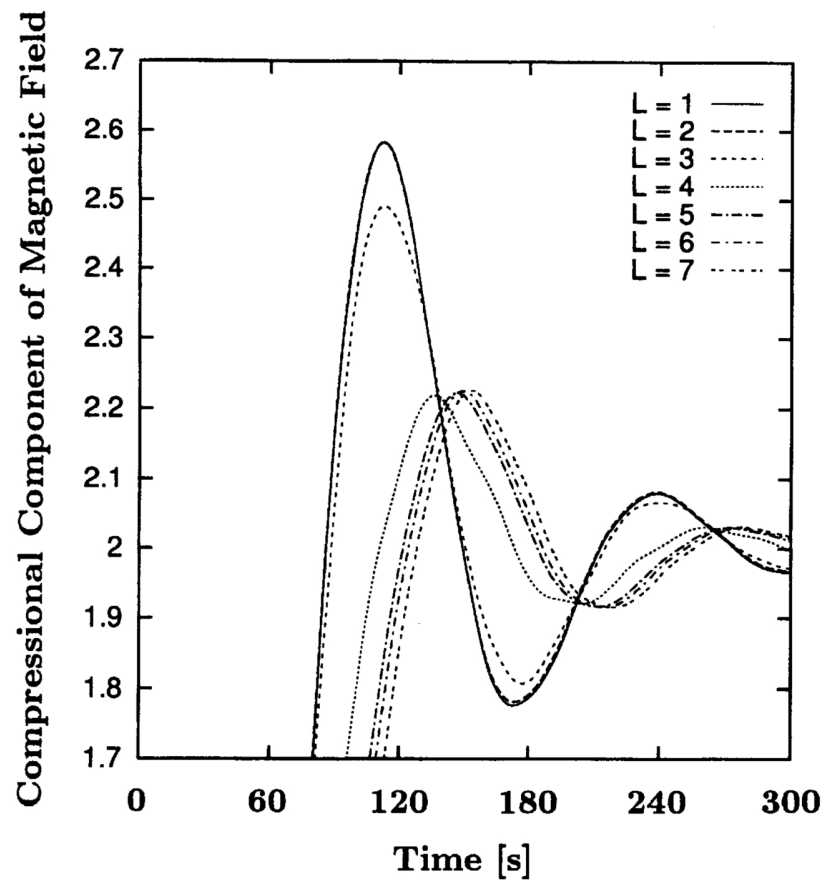

Fig. 3. Superposition of the waveforms at $L=1,2,3,4,5,6$ and 7 displayed in Fig. 2. The relative phase between the oscillations at the earth's surface and another $L$ shell increases monotonically with $L$.

Table 1. First six poles estimated from the waveform at $L=1$ in Fig. 2. Given in the parentheses are true poles in case of $L_{n}=7$.

\begin{tabular}{cccc}
\hline Number & Pole $\left(\hat{p} / T\left[\mathrm{~s}^{-1}\right]\right)$ & Number & Pole $\left(\hat{p} / T\left[\mathrm{~s}^{-1}\right]\right)$ \\
\hline 1 & $-0.0155563+0.0496700 i$ & 4 & $-0.0439134+0.4811785 i$ \\
& $(-0.0155562+0.0496697 i)$ & & $(-0.0439205+0.4811292 i)$ \\
2 & $-0.0111909+0.2039679 i$ & 5 & $-0.0434882+0.5836685 i$ \\
& $(-0.0111910+0.2039682 i)$ & & $(-0.0435528+0.5836456 i)$ \\
3 & $-0.0236265+0.3456596 i$ & 6 & $-0.0370084+0.7004937 i$ \\
& $(-0.0236220+0.3456620 i)$ & & $(-0.0370002+0.7005714 i)$ \\
\hline
\end{tabular}

Table 2. First six poles estimated from the waveform at $L=2$ in Fig. 2 .

\begin{tabular}{cccc}
\hline Number & Pole $\left(\hat{p} / T\left[\mathrm{~s}^{-1}\right]\right)$ & Number & Pole $\left(\hat{p} / T\left[\mathrm{~s}^{-1}\right]\right)$ \\
\hline 1 & $-0.0155561+0.0496698 i$ & 4 & $-0.0437240+0.4812510 i$ \\
2 & $-0.0111911+0.2039682 i$ & 5 & $-0.0448777+0.5849166 i$ \\
3 & $-0.0236233+0.3456746 i$ & 6 & $-0.0374165+0.7007380 i$ \\
\hline
\end{tabular}

of damping factor and angular frequency are represented by $\widehat{\alpha}$ and $\widehat{\omega}$, respectively, the pole is estimated with $\widehat{p}=-\widehat{\alpha}+i \widehat{\omega}$. The first six poles estimated from the waveforms at $L=1,2$, 3, 4, 5, 6 and 7 in Fig. 2 are listed in Tables 1, 2, 3, 4, 5, 6 and 7, respectively, where they are numbered in order of the angular frequency. Given in the parentheses in Table 1 are true poles in case of $L_{n}=7$. It is found in these tables that the estimates of the first six poles agree 
Table 3. First six poles estimated from the waveform at $L=3$ in Fig. 2 .

\begin{tabular}{cccc}
\hline Number & Pole $\left(\hat{p} / T\left[\mathrm{~s}^{-1}\right]\right)$ & Number & Pole $\left(\widehat{p} / T\left[\mathrm{~s}^{-1}\right]\right)$ \\
\hline 1 & $-0.0155562+0.0496698 i$ & 4 & $-0.0439611+0.4811041 i$ \\
2 & $-0.0111910+0.2039682 i$ & 5 & $-0.0435572+0.5834542 i$ \\
3 & $-0.0236211+0.3456621 i$ & 6 & $-0.0370886+0.7004362 i$ \\
\hline
\end{tabular}

Table 4. First six poles estimated from the waveform at $L=4$ in Fig. 2 .

\begin{tabular}{cccc}
\hline Number & Pole $\left(\hat{p} / T\left[\mathrm{~s}^{-1}\right]\right)$ & Number & Pole $\left(\widehat{p} / T\left[\mathrm{~s}^{-1}\right]\right)$ \\
\hline 1 & $-0.0155564+0.0496699 i$ & 4 & $-0.0439011+0.4811836 i$ \\
2 & $-0.0111910+0.2039682 i$ & 5 & $-0.0435253+0.5837286 i$ \\
3 & $-0.0236222+0.3456598 i$ & 6 & $-0.0368607+0.7004102 i$ \\
\hline
\end{tabular}

Table 5. First six poles estimated from the waveform at $L=5$ in Fig. 2 .

\begin{tabular}{cccc}
\hline Number & Pole $\left(\hat{p} / T\left[\mathrm{~s}^{-1}\right]\right)$ & Number & Pole $\left(\widehat{p} / T\left[\mathrm{~s}^{-1}\right]\right)$ \\
\hline 1 & $-0.0155561+0.0496695 i$ & 4 & $-0.0438571+0.4811512 i$ \\
2 & $-0.0111908+0.2039684 i$ & 5 & $-0.0434821+0.5836094 i$ \\
3 & $-0.0236249+0.3456633 i$ & 6 & $-0.0377988+0.7005939 i$ \\
\hline
\end{tabular}

Table 6. First six poles estimated from the waveform at $L=6$ in Fig. 2 .

\begin{tabular}{cccc}
\hline Number & Pole $\left(\hat{p} / T\left[\mathrm{~s}^{-1}\right]\right)$ & Number & Pole $\left(\hat{p} / T\left[\mathrm{~s}^{-1}\right]\right)$ \\
\hline 1 & $-0.0155562+0.0496699 i$ & 4 & $-0.0438784+0.4810856 i$ \\
2 & $-0.0111913+0.2039682 i$ & 5 & $-0.0435109+0.5834717 i$ \\
3 & $-0.0236229+0.3456620 i$ & 6 & $-0.0380847+0.7007094 i$ \\
\hline
\end{tabular}

Table 7. First six poles estimated from the waveform at $L=7$ in Fig. 2 .

\begin{tabular}{cccc}
\hline Number & Pole $\left(\hat{p} / T\left[\mathrm{~s}^{-1}\right]\right)$ & Number & Pole $\left(\hat{p} / T\left[\mathrm{~s}^{-1}\right]\right)$ \\
\hline 1 & $-0.0155561+0.0496699 i$ & 4 & $-0.0438998+0.4811354 i$ \\
2 & $-0.0111909+0.2039682 i$ & 5 & $-0.0435787+0.5836236 i$ \\
3 & $-0.0236254+0.3456630 i$ & 6 & $-0.0381032+0.7013712 i$ \\
\hline
\end{tabular}

well with the true poles. Other poles corresponding to higher frequencies were poorly estimated because their corresponding oscillations were likely to have much smaller amplitudes. Further, we could not discover any damped sinusoid which has a frequency less than $0.1 \mathrm{~Hz}$ but has no corresponding pole. Thus, it is verified that the oscillations found in Fig. 2 are due to the poles of the system under consideration. Figure 4 exhibits a superposition of the estimated damped sinusoids at $L=1,2,3,4,5,6$ and 7 corresponding to the first pole. It is also seen from this figure that the relative phase increases monotonically with $L$, although there exists little phase lag between $L=1$ and $L=2$.

\subsection{Impulse-like disturbances}

In this subsection we will consider the transient response of the inner equatorial magnetosphere to earthward propagating impulse-like MHD disturbances. The impulse-like disturbance 


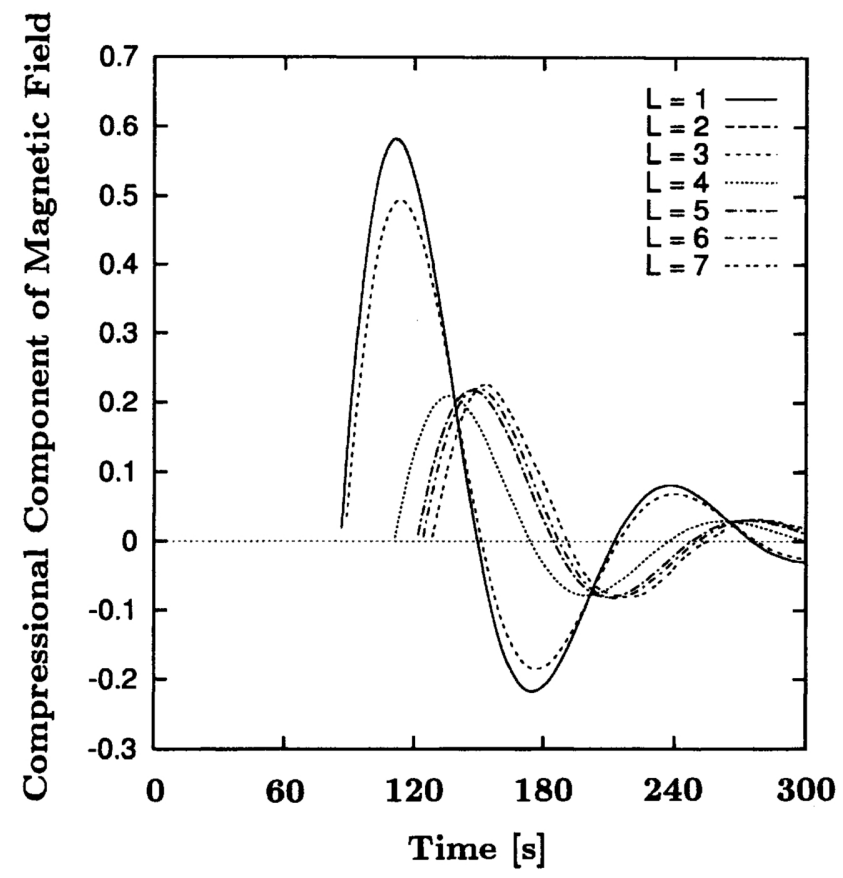

Fig. 4. Superposition of the damped sinusoids corresponding to the first pole which are estimated from the waveforms simulated at $L=1,2,3,4,5,6$ and 7 displayed in Fig. 2. There exists little phase lag between $L=1$ and $L=2$.

incident on the outer boundary of the inner magnetosphere, which begins at $\tau=0$, is represented by

$$
\delta B_{\text {in }}(\tau)=13.8571 \delta B_{\max } \tau \mathrm{e}^{-5 \tau} \cos \tau
$$

with a maximum amplitude $\delta B_{\max }$. Then, we have

$$
\delta \widehat{B}_{\text {in }}(p)=6.92855 \delta B_{\max }\left[\frac{1}{(p+5+i)^{2}}+\frac{1}{(p+5-i)^{2}}\right]
$$

by Laplace-transforming $\delta B_{\text {in }}(\tau)$. Note that the characteristic time scale $T$ is an effective duration of the disturbance. Such a disturbance has been used in Allan et al. $(1985,1986)$.

Figure 5 shows transient waveforms (solid lines) of the compressional component $\delta B_{x}$ of the perturbation magnetic field, which are normalized by $\delta B_{\max }\left(\delta B_{\max }=1\right)$, simulated at the earth's surface $(L=1)$ for the incident impulse-like disturbances (dashed lines) at $L=7$ with $T=10,30,60,120,300$ and $600 \mathrm{~s}$. Here, the inner magnetosphere has also no strong Alfvén velocity gradient at its outer boundary $\left(V_{n}=\mathrm{AS}_{1}\left(L_{n}\right)\right)$. The arrows in this figure indicate the travel time of wave across the $L$ range of 1 to 7 . The small-amplitude high-frequency noise in case of $T=10 \mathrm{~s}$ is due to a numerical error. In general, as seen from Fig. 5, the oscillation arising from the cavity resonance all over the inner magnetosphere is also most predominant. However, its amplitude becomes smaller as $T$ grows large, and it is negligibly small for $T$ greater than several times of eigenperiod of the resonance. On the other hand, when $T$ is relatively small (e.g., $T \lesssim 10 \mathrm{~s}$ ), the oscillations due to the trapped resonances are outstanding. We have found that these characteristics are independent of whether or not the inner magnetosphere has a strong Alfvén velocity gradient at its outer boundary (not shown). The impulse response of the system 

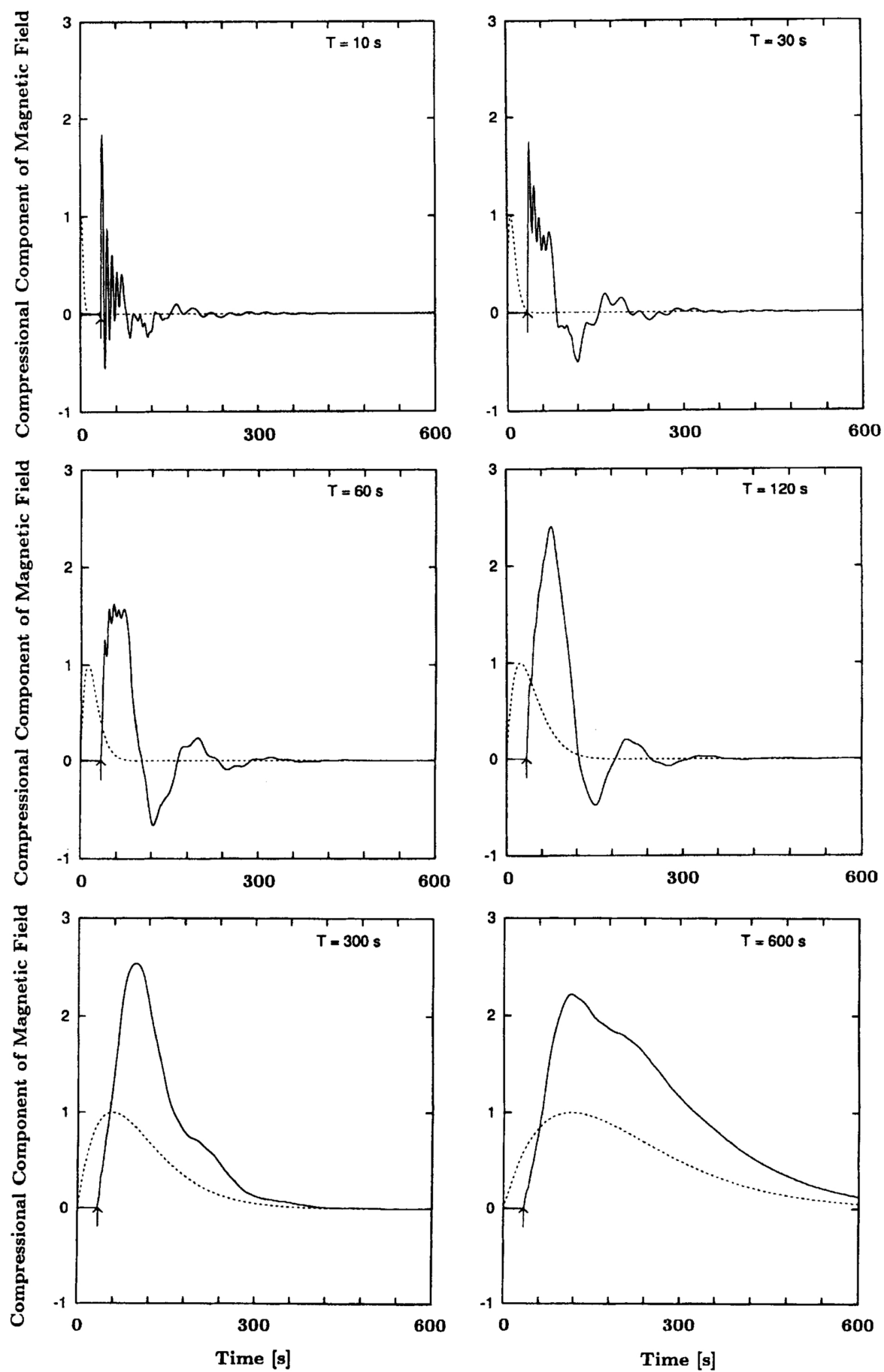

Fig. 5. Transient waveforms (solid lines) of the compressional component $\delta B_{x}$ simulated at $L=1$ for incident impulse-like disturbances (dashed lines) at $L=7$ with $T=10,30,60,120,300$ and $600 \mathrm{~s}$ when the inner magnetosphere has no strong Alfvén velocity gradient at its outer boundary $\left(V_{n}=\operatorname{AS}_{1}\left(L_{n}\right)\right)$. Here, model 1 $\left(\mathrm{AS}_{1}\right)$ is employed for the altitude distribution of $V_{\mathrm{A}}$, and the values of $\sigma_{\mathrm{E}}=0.001 \mathrm{~S} / \mathrm{m}$ and $\Sigma_{\mathrm{C}}=1 \mathrm{~S}$ are used. 
under consideration at $L=1$ would become something analogous to the transient waveform for $T=10 \mathrm{~s}$ displayed in Fig. 5 .

Sampling $\delta B_{x}$ with an interval of $\Delta \tau=0.02$ from the instant when the disturbance arrives and denoting the samples by $s_{n}(n=0,1, \ldots)$, we also estimate the parameters using Eq. (10). The first eight poles estimated from the waveform at $L=1$ in case of $L_{n}=7$ and $T=60$ s are listed in Table 8, where they are also numbered in order of the angular frequency. Also given in the parentheses in Table 8 are true poles in case of $L_{n}=7$. While the seventh and eighth poles as well as the first six ones are well estimated in this case, this is because the oscillations

Table 8. First eight poles estimated from the waveform at $L=1$ for the incident impulse-like disturbance with $T=60 \mathrm{~s}$ at $L=7$. Given in the parentheses are true poles in case of $L_{n}=7$.

\begin{tabular}{cccc}
\hline Number & Pole $\left(\hat{p} / T\left[\mathrm{~s}^{-1}\right]\right)$ & Number & Pole $\left(\hat{p} / T\left[\mathrm{~s}^{-1}\right]\right)$ \\
\hline 1 & $-0.0155532+0.0496687 i$ & 5 & $-0.0436578+0.5839719 i$ \\
& $(-0.0155562+0.0496697 i)$ & & $(-0.0435528+0.5836456 i)$ \\
2 & $-0.0111905+0.2039669 i$ & 6 & $-0.0369240+0.7007136 i$ \\
& $(-0.0111910+0.2039682 i)$ & & $(-0.0370002+0.7005714 i)$ \\
3 & $-0.0236323+0.3456767 i$ & 7 & $-0.0418014+0.7847724 i$ \\
& $(-0.0236220+0.3456620 i)$ & & $(-0.0415499+0.7846806 i)$ \\
4 & $-0.0437661+0.4813562 i$ & 8 & $-0.0451954+0.8760736 i$ \\
& $(-0.0439205+0.4811292 i)$ & & $(-0.0449674+0.8766771 i)$ \\
\hline
\end{tabular}

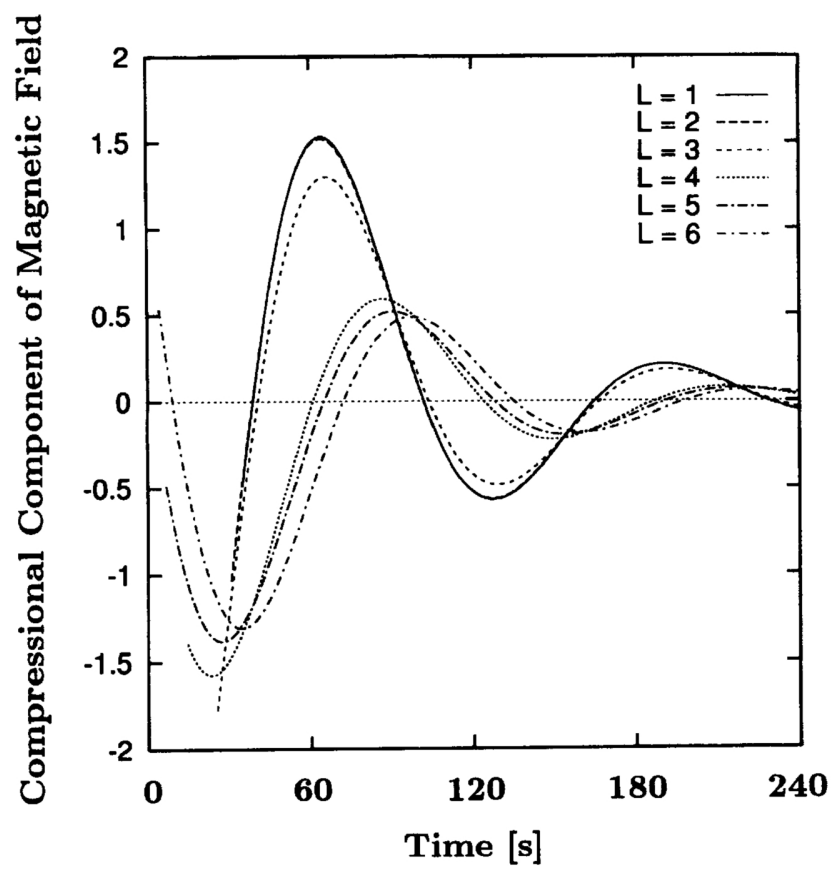

Fig. 6. Superposition of the damped sinusoids corresponding to the first pole which are estimated from the waveforms simulated at $L=1,2,3,4,5$ and 6 for the incident impulse-like disturbance at $L=7$ with $T=60 \mathrm{~s}$ when the inner magnetosphere has no strong Alfvén velocity gradient at its outer boundary $\left(V_{n}=\operatorname{AS}_{1}\left(L_{n}\right)\right)$. Here, model $1\left(\mathrm{AS}_{1}\right)$ is employed for the altitude distribution of $V_{\mathrm{A}}$, and the values of $\sigma_{\mathrm{E}}=0.001 \mathrm{~S} / \mathrm{m}$ and $\Sigma_{\mathrm{C}}=1 \mathrm{~S}$ are used. There exists little phase lag between $L=1$ and $L=2$. 
corresponding to those poles have larger amplitudes. Although the first eight poles are well estimated for any smaller $L$ shell, the estimation of some poles becomes poorer as $L$ grows large. At $L=7$, especially, even the first pole failed in an estimation. This means that assuming Eq. (10) for the samples starting at the instant when the disturbance arrives is not valid for larger $L$ shells. However, when the samples starting later were used, a good estimation was made for any larger $L$ shell. Figure 6 exhibits a superposition of the estimated damped sinusoids at $L=1,2,3,4,5$ and 6 corresponding to the first pole. It is also confirmed from this figure that the relative phase increases monotonically with $L$.

\section{Discussion}

In order to give an answer to the problem whether the cavity-mode type resonance of the inner magnetosphere is the sole cause of the equatorial Pi2 pulsations, we present two examples of low-latitude and equatorial Pi2's observed on the ground and compare those pulsations with the waveforms simulated at $L=1$. Figure 7 is the first example observed at Kuju (KUJ) ground station located at $L \simeq 1.2$ and $\mathrm{LT}=\mathrm{UT}+9$ on Jan. 20, 1991. Shown in this figure are raw $H$ component geomagnetic data sampled every $3 \mathrm{~s}$ as well as smoothed and residual data processed by the PCP filter with $\mu=10$ corresponding to the cutoff frequency of $2.757 \mathrm{mHz}$ for $3 \mathrm{~s}$ sampling (Itonaga, 1997). A Pi2 pulsation and a positive bay disturbance with a rapid change began around 15:54 UT. This bay disturbance may be analogous to the step-like disturbance handled in the last section. The vertical arrows in Fig. 7 indicate the positions of knots of the piecewise cubic polynomial in the neighborhood of the rapid change. As for some more explanations of this example refer to Itonaga and Kitamura (1994). Since the interval between two adjacent knots indicated by the third and fourth arrows is $150.0 \mathrm{~s}$, this event might be comparable with the waveform for $T=120 \mathrm{~s}$ in Fig. 1. It is seen at a glance that the waveform of the Pi2 in Fig. 7 is much more complicated than the simulated one in Fig. 1. To confirm this, applying Eq. (10) to the residual data in the interval of 15:54:09 UT to 16:00:21 UT $(N=125)$, we estimated the parameters. The estimated poles are listed in Table 9 , where they are numbered in order of

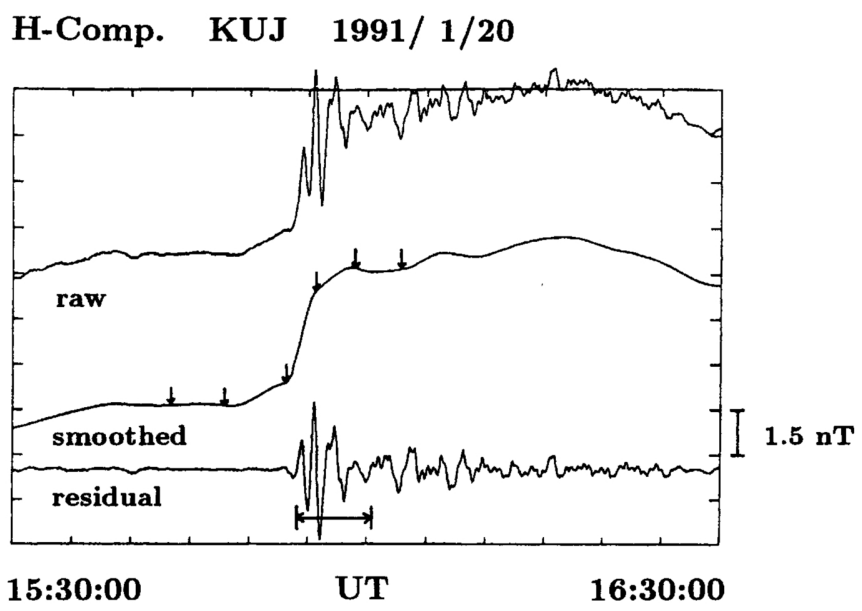

Fig. 7. Raw $H$-component geomagnetic data observed at KUJ on Jan. 20, 1991 and sampled every $3 \mathrm{~s}$ as well as smoothed and residual data processed by the PCP filter with $\mu=10$. The vertical arrows indicate the positions of knots of the piecewise cubic polynomial in the neighborhood of the rapid change. The horizontal left-right arrow represents the interval of 15:54:09 UT to 16:00:21 UT used in the parameter estimation. 
Table 9. Poles estimated from the residual data at KUJ in the interval of 15:54:09 UT to 16:00:21 UT of Jan. 20, 1991.

\begin{tabular}{cccc}
\hline Number & Pole $\left[\mathrm{s}^{-1}\right]$ & Number & Pole $\left[\mathrm{s}^{-1}\right]$ \\
\hline 1 & $-0.0109869+0.0486861 i$ & 5 & $-0.0019173+0.1742481 i$ \\
2 & $-0.0147909+0.0589258 i$ & 6 & $-0.0052467+0.2526937 i$ \\
3 & $-0.0150723+0.1088089 i$ & 7 & $-0.0049937+0.3047414 i$ \\
4 & $-0.0068465+0.1444948 i$ & & \\
\hline
\end{tabular}

H-Comp. HUA $1986 / 2 / 1$

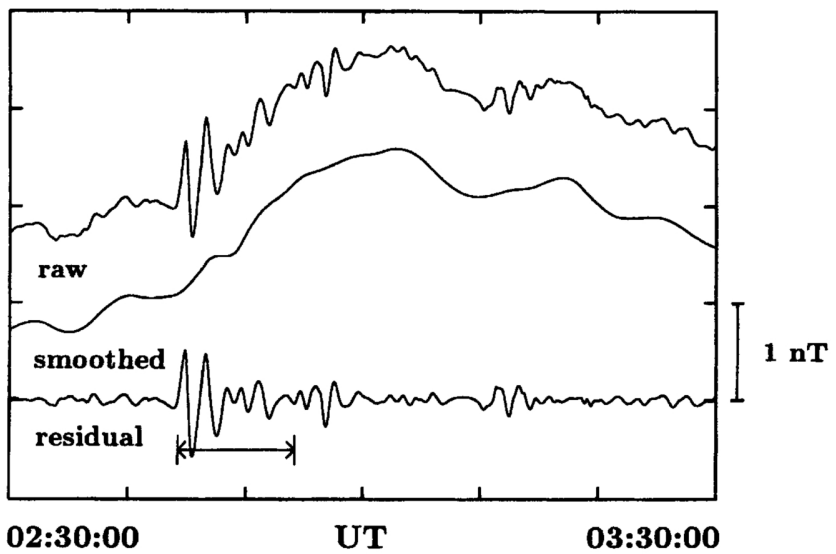

Fig. 8. Raw $H$-component geomagnetic data observed at HUA on Feb. 1, 1986 and sampled every $3 \mathrm{~s}$ as well as smoothed and residual data processed by the PCP filter with $\mu=9$. The horizontal left-right arrow represents the interval of 02:44:12 UT to 02:54:09 UT used in the parameter estimation.

the angular frequency. The oscillations due to the first four poles were predominant in power. Although some of the poles listed in Table 9 might arise from the cavity-mode type resonances, it would be impossible that all of these poles have origin in such resonances. In fact, lower and nearby frequencies of the first four poles are unlikely to be reproduced by only an appropriate regulation of the parameters which determine the altitude distribution of $V_{\mathrm{A}}$ used in the system under consideration.

Figure 8 is the second example observed at Huancayo (HUA) ground station located at the dip equator and LT $=\mathrm{UT}-5$ on Feb. 1, 1986. Displayed in this figure are raw $H$-component geomagnetic data sampled every $3 \mathrm{~s}$ as well as smoothed and residual data processed by the PCP filter with $\mu=9$ corresponding to the cutoff frequency of $3.373 \mathrm{mHz}$ for $3 \mathrm{~s}$ sampling (Itonaga, 1996). A Pi2 pulsation and a positive bay disturbance with a gradual change began around 02:44 UT. Applying Eq. (10) to the residual data in the interval of 02:44:12 UT to 02:54:09 UT $(N=200)$, we also estimated the parameters. The estimated poles are listed in Table 10, where they are also numbered in order of the angular frequency. The oscillations due to the first three poles dominanted in power. Although some of the poles listed in Table 10 might originate in the cavity-mode type resonances, it would be also impossible that all of these poles are caused by such resonances.

As argued by Takahashi et al. $(1992,1995)$, the cavity-mode type resonance of the inner magnetosphere will be surely a cause of the equatorial Pi2's. However, the above two examples suggest that some more mechanisms may take part in the occurrence of the Pi2's, because the 
Table 10. Poles estimated from the residual data at HUA in the interval of 02:44:12 UT to 02:54:09 UT of Feb. $1,1986$.

\begin{tabular}{cccc}
\hline Number & Pole $\left[\mathrm{s}^{-1}\right]$ & Number & Pole $\left[\mathrm{s}^{-1}\right]$ \\
\hline 1 & $-0.0030029+0.0469620 i$ & 4 & $-0.0023056+0.1087494 i$ \\
2 & $-0.0051321+0.0669737 i$ & 5 & $-0.0082423+0.1292269 i$ \\
3 & $-0.0040414+0.0808168 i$ & 6 & $-0.0095955+0.1931187 i$ \\
\hline
\end{tabular}

lower and nearby frequencies of some poles are unlikely to be brought about by only the simple cavity-mode type resonance. As for equatorial Pi2 events observed within 3 hours of midnight, Takahashi et al. (1992) reported that the relative phase between the compressional component at the satellite and the $H$ component at the earth's surface is either near zero or near $180^{\circ}$, with the $\sim 180^{\circ}$ phase lag observed only when the satellite is located at $L>3$. Such an observational result supports the above suggestion. If the cavity-mode type resonance were the sole cause, the relative phase between the oscillations at the earth's surface and another $L$ shell would increase monotonically with $L$, as found in Figs. 4 and 6 .

The real parts of the first four poles in Table 10 are considerably smaller in magnitude than those of the first three poles in Table 9. Then, if these poles arise from the cavity-mode type resonance, some strong Alfvén velocity gradient may have to be taken into account at the outer boundary of the inner magnetosphere. Figure 9 shows a comparison of the transient waveform (solid lines) at $L=1$ with those (dashed lines) at $L=2,3,4,5,6$ and 7 in case of the inner magnetosphere having a strong positive Alfvén velocity gradient at its outer boundary $\left(V_{n}=10 \times \mathrm{AS}_{1}\left(L_{n}\right)\right)$. Here, $L_{n}=7$ and the incident disturbance is given by Eq. (8) with $T=60$ $\mathrm{s}$ and $\delta B_{\max }=1$. In this case the compressional oscillations have larger amplitudes and long-lived natures. While the compressional oscillation originating in the first pole is most predominant at any $L$ shell, the oscillation due to the second pole is also outstanding at $L \gtrsim 4$ except in the vicinity of $L=7$ (cf. Fig. 6 of paper 1). As expected from the case (a) of Fig. 6 in paper 1, the magnetic energy of the compressional oscillation due to the first pole dominates overwhelmingly within the plasmasphere. This is also confirmed in Fig. 10 exhibiting a superposition of the estimated damped sinusoids at $L=1,2,3,4,5,6$ and 7 corresponding to the first pole. The estimating procedure similar to that in Fig. 4 was also used in Fig. 10. It is found in Fig. 10 that the relative phase also increases monotonically with $L$, although there exists little phase lag between $L=1$ and $L=2$. Thus, the phase structure presented by Takahashi et al. (1992) is not reproduced in case of $V_{n}=10 \times \mathrm{AS}_{1}\left(L_{n}\right)$ either.

Figure 11 displays a comparison of the transient waveform (solid lines) at $L=1$ with those (dashed lines) at $L=2,3,4,5,6$ and 7 in case of the inner magnetosphere having a strong negative Alfvén velocity gradient at its outer boundary $\left(V_{n}=\mathrm{AS}_{1}\left(L_{n}\right) / 10\right)$. Here, $L_{n}=7$ and the incident disturbance is also given by Eq. (8) with $T=60 \mathrm{~s}$ and $\delta B_{\max }=1$. When $V_{n}=\mathrm{AS}_{1}\left(L_{n}\right) / 10$, the magnetic energy is slowly injected into the inner magnetosphere and the compressional oscillations have smaller amplitudes. However, the oscillations have long-lived natures in this case as well as in case of $V_{n}=10 \times \mathrm{AS}_{1}\left(L_{n}\right)$. Exhibited in Fig. 12 is a superposition of the residuals obtained by processing the transient waveforms in Fig. 11 with the PCP filter having the cutoff frequency of $\sim 5 \mathrm{mHz}$. In this figure only the residuals at $L=1,3,4$ and 7 are plotted from the instant when the disturbance arrives. When $V_{n}=\mathrm{AS}_{1}\left(L_{n}\right) / 10$, in contrast to the cases of $V_{n}=\operatorname{AS}_{1}\left(L_{n}\right)$ and $V_{n}=10 \times \mathrm{AS}_{1}\left(L_{n}\right)$, the relative phase between the oscillations at the earth's surface and another $L$ shell is near zero and near $180^{\circ}$ at $L<3.867$ and $L>3.867$, respectively. Here, the node of the steady-state oscillation in the case (a) of Fig. 8 in paper 1 is located at $L=3.867$ and the phase jump of $\sim \pi$ occurs there. Thus, if there exists a strong negative Alfvén velocity gradient at the outer boundary of the inner magnetosphere, such a phase structure as reported 

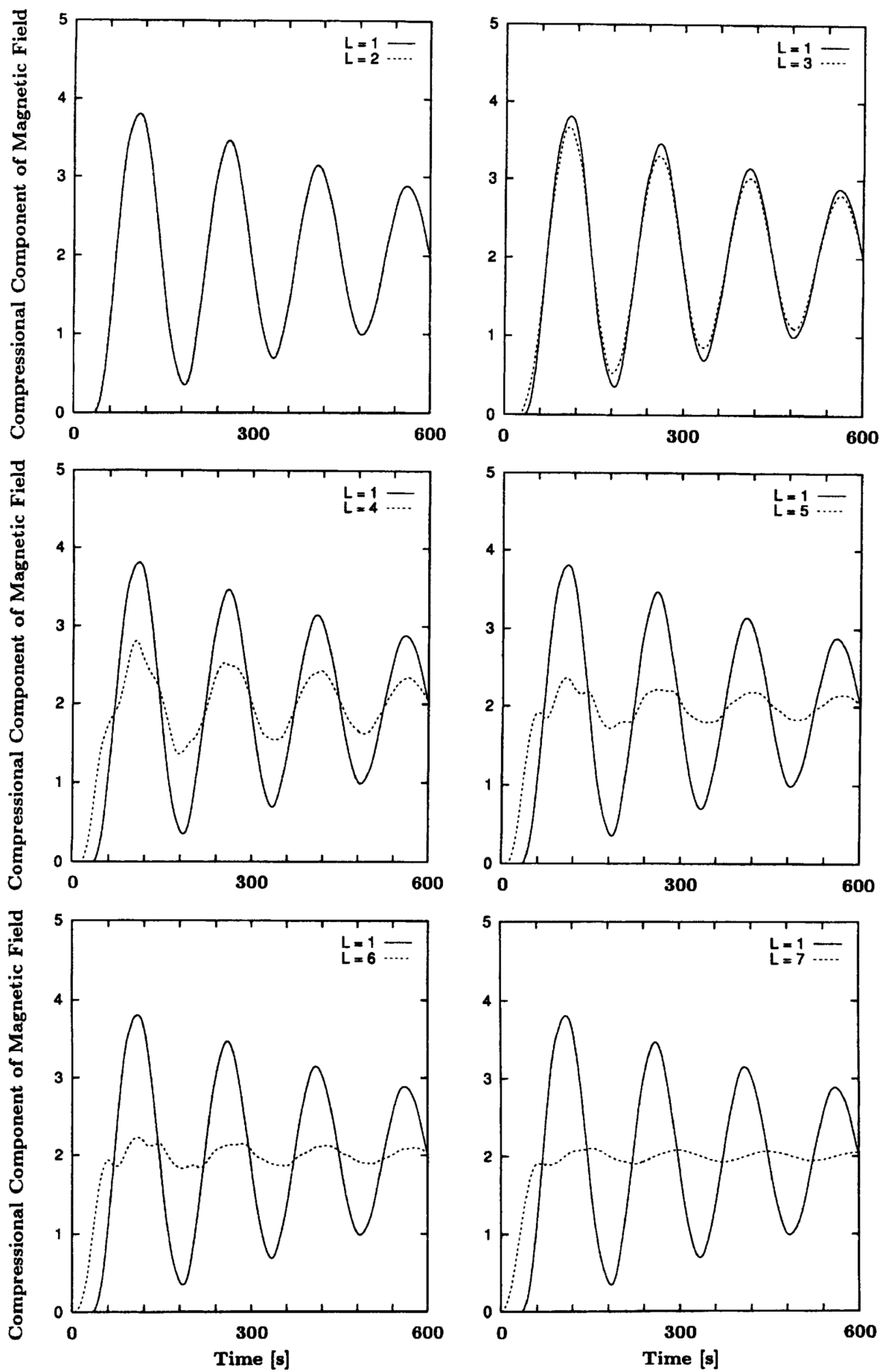

Fig. 9. Same as Fig. 2 except in case of the inner magnetosphere having a strong positive Alfvén velocity gradient at its outer boundary $\left(V_{n}=10 \times \operatorname{AS}_{1}\left(L_{n}\right)\right)$. 


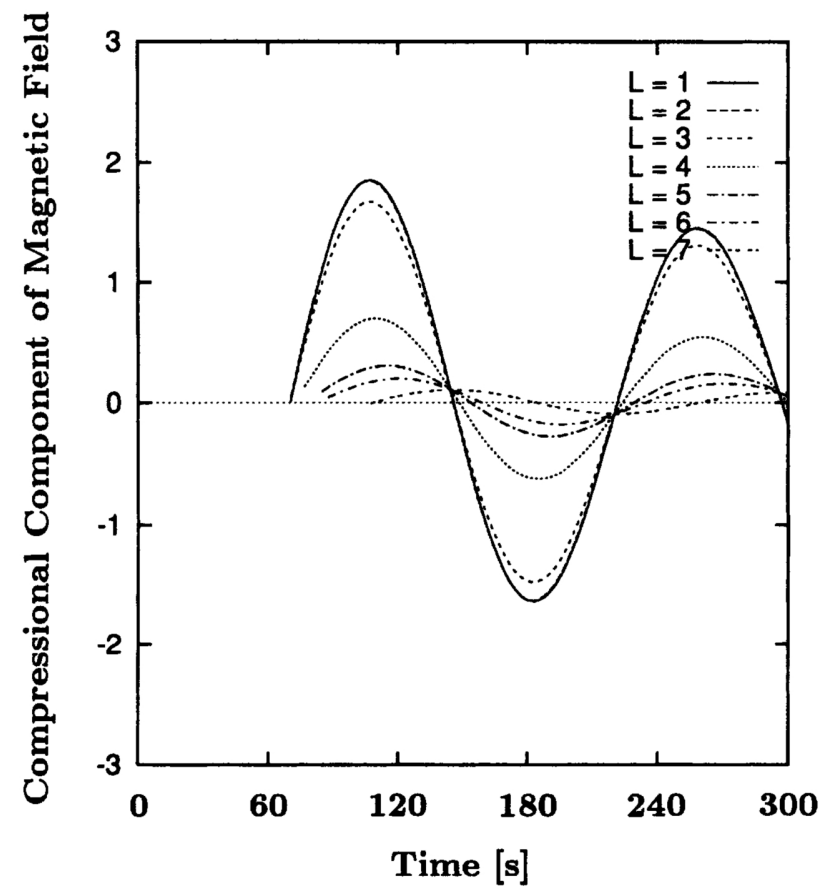

Fig. 10. Superposition of the damped sinusoids corresponding to the first pole which are estimated from the waveforms simulated at $L=1,2,3,4,5,6$ and 7 displayed in Fig. 9. There exists little phase lag between $L=1$ and $L=2$.

by Takahashi et al. (1992) may be observed also in the cavity-mode oscillation.

The quasi-steady-state oscillations would have phase structures similar to those of steadystate oscillations and so could bring about such a phase structure as presented by Takahashi et al. (1992). Then, some constituents of the equatorial Pi2's might be not cavity-mode oscillations but quasi-steady-state compressional oscillations forced by some damped sinusoidal waves incident on the outer boundary of the inner magnetosphere. Supposed as a candidate for the damped sinusoidal wave will be the earthward propagating compressional wave emitted from a large scale oscillating current wedge which is transiently formed in association with the cross-tail current disruption (Baumjohann and Glassmeier, 1984). Such a compressional wave accompanies the bay disturbance launched by a large scale quasi-static current wedge. As seen from Fig. 5, the impulsive release of magnetic energy is unlikely to produce such a bay disturbance as observed on the ground. Thus, the bay disturbance (or at least its initial part) would be due to the quasi-steplike release of magnetic energy. The inner magnetosphere might have a strong negative Alfvén velocity gradient at its outer boundary in the example of Fig. 8.

Although the lower and nearby frequencies of the low-latitude and equatorial Pi2's observed on the ground are unlikely to be caused by only the one-dimensional cavity-mode type resonance, some three-dimensional resonance might be able to yield such frequencies. A limit (or discontinuity) of the strong gradient at the outer boundary has been considered in the present study. Such a gradient is much stronger than any realistic gradient. Nevertheless, the absolute value of real part of the first pole of the system under consideration is as small as the damping factors of the damped sinusoids with lower frequencies in the second example mentioned above. In case of the three-dimensional cavity-mode type resonance, since the energy escapes from the resonance 

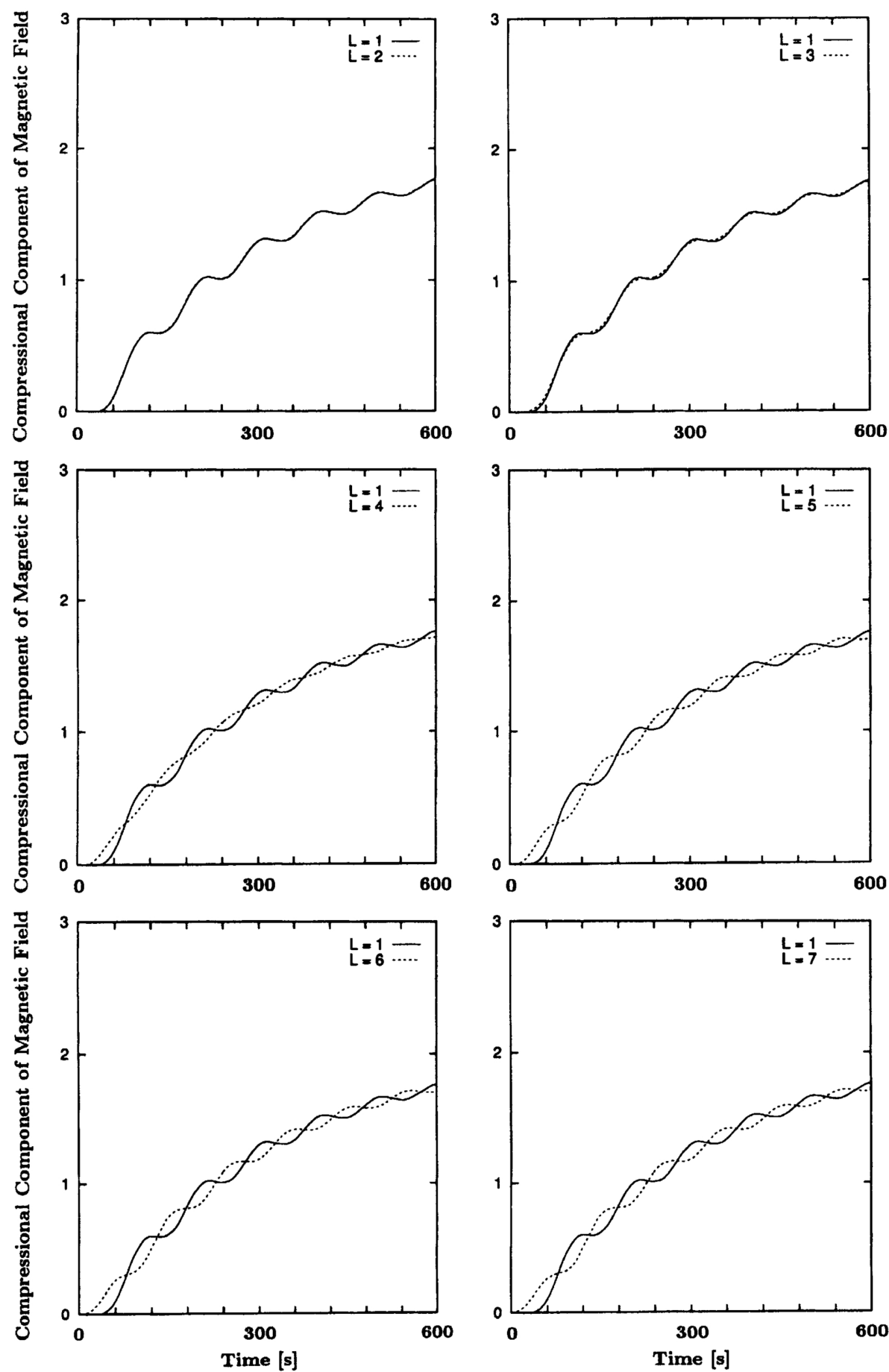

Fig. 11. Same as Fig. 2 except in case of the inner magnetosphere having a strong negative Alfvén velocity gradient at its outer boundary $\left(V_{n}=\operatorname{AS}_{1}\left(L_{n}\right) / 10\right)$. 


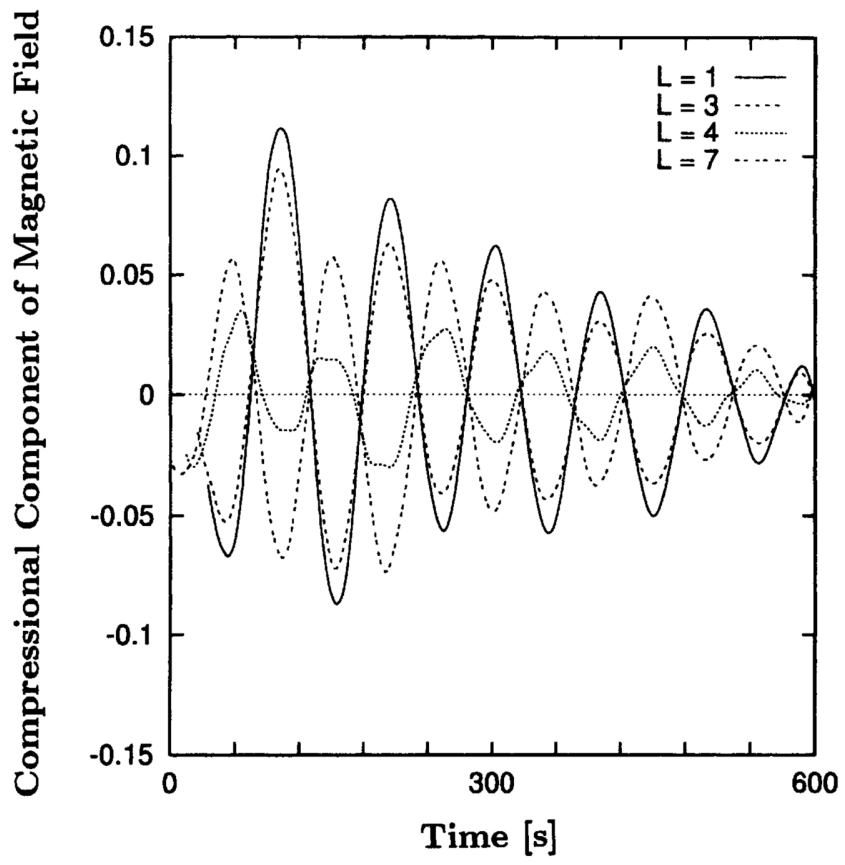

Fig. 12. Superposition of the residuals at $L=1,3,4$ and 7 obtained by processing the transient waveforms displayed in Fig. 11 with the PCP filter having the cutoff frequency of $\sim 5 \mathrm{mHz}$.

region through its two-dimensional outer boundary, the cavity-mode oscillation will be damped more quickly. Then, for the three-dimensional resonance it becomes an issue how and where an extremely strong Alfvén velocity gradient making the damping factor of cavity-mode oscillation as small as those of the damped sinusoids in the second example can be formed. Further, the great majority of the equatorial Pi2's in the inner magnetosphere are observed within 3 hours of midnight (Takahashi et al., 1992). This contradicts the properties of the three-dimensional cavity-mode type resonance. If the resonance were the sole cause of the Pi2's, the energy would be quickly distributed to all local times, because there are no obvious local time boundaries to prevent azimuthal propagation of compressional waves, and the Pi2's could be observed all over the inner magnetosphere. Thus, unless there exist any physical mechanisms which form the extremely strong Alfvén velocity gradient and prevent the azimuthal propagation, the threedimensional resonance will be not so much promising as a cause of the Pi2's.

To make a close examination of the validity of the one-dimensional model used in the present study, we need to estimate the effect of spatial spread of the reflected MHD compressional wave by the spherical solid earth on the transient response of the inner magnetosphere. It will be quite difficult to perform such an estimation quantitatively in the general case of three-dimensional propagation. However, it is possible to examine two-dimensional transient response of the inner magnetosphere at the magnetic equator using a cylindrical model where the ambient magnetic field $\mathbf{B}$ is parallel to the axis of the cylinder. Through such an examination we can find a clue to the estimation of the effect of spatial spread of the reflected wave. The two-dimensional transient response is a future subject of investigation following the present study. 


\section{Summary}

Using a model of altitude distribution of the Alfvén speed $V_{\mathrm{A}}$, we considered one-dimensional transient response of the inner magnetosphere at the magnetic equator to earthward propagating impulse- and step-like MHD disturbances. We directly simulated waveforms of transient compressional oscillations due to these disturbances at some $L$ shells by a numerical inversion of the Laplace transform with orthonormal Laguerre functions. Through in detail examining these simulated waveforms in connection with the observational results obtained by Kyushu University as well as those by Takahashi et al. $(1992,1995)$, then, we tackled the problem whether the cavity-mode type resonance was the sole cause of the equatorial Pi2 pulsations.

It was verified that the compressional oscillations are due to the poles of the system under consideration. The oscillation arising from the cavity resonance all over the inner magnetosphere is most dominant. However, its amplitude becomes smaller as the characteristic time scale $T$ of an incident disturbance (a rise time of the step-like disturbance or an effective duration of the impulse-like disturbance) grows large, and it is negligibly small for $T$ greater than several times of eigenperiod of the resonance. On the other hand, when $T$ is relatively small (e.g., $T \lesssim 10 \mathrm{~s}$ ), the oscillations due to the cavity resonances trapped around the trough in $V_{\mathrm{A}}$ are outstanding. These characteristics are independent of whether or not the inner magnetosphere has a strong gradient of $V_{\mathrm{A}}$ at its outer boundary. When the inner magnetosphere has a strong positive gradient of $V_{\mathrm{A}}$ at its outer boundary, the compressional oscillations have larger amplitudes and long-lived natures. Further, the magnetic energy of the oscillation arising from the cavity resonance all over the inner magnetosphere dominates overwhelmingly within the plasmasphere. On the other hand, when the inner magnetosphere has a strong negative gradient of $V_{\mathrm{A}}$ at its outer boundary, the magnetic energy is slowly injected into the inner magnetosphere and the compressional oscillations have smaller amplitudes and long-lived natures. It was also found that the relative phase between the cavity-mode oscillations all over the inner magnetosphere at the earth's surface and another $L$ shell increases monotonically with $L$ when the inner magnetosphere has no strong gradient or a strong positive gradient of $V_{\mathrm{A}}$ at its outer boundary. However, the relative phase is nearly zero and nearly $180^{\circ}$ inside and outside a specific $L$ shell, respectively, when the inner magnetosphere has a strong negative gradient at its outer boundary.

The one-dimensional cavity-mode type resonance of the inner magnetosphere is certainly a cause of the equatorial Pi2's. However, some more mechanisms may take part in the occurrence of the Pi2's because lower and nearby frequencies of low-latitude and equtorial Pi2's observed on the ground are unlikely to be brought about by only the simple cavity-mode type resonance. The quasi-steady-state oscillations would have phase structures similar to those of steady-state oscillations and so could yield such a phase structure as reported by Takahashi et al. (1992). Then, some constituents of the Pi2's may be not cavity-mode oscillations but quasi-steady-state oscillations forced by some damped sinusoidal waves incident on the outer boundary of the inner magnetosphere. A candidate for the damped sinusoidal wave will be the earthward propagating compressional wave emitted from a large scale oscillating current wedge which is transiently formed in association with the cross-tail current disruption.

The authors would like to express their thanks to S. Fujita, T.-I. Kitamura, K. Takahashi and T. Watanabe for their helpful discussions. This work was partly supported by the Grant-in-Aid for Scientific Research (C), No. 05640488, from The Ministry of Education, Science and Culture.

\section{REFERENCES}

Allan, W., S. P. White, and E. M. Poulter, Magnetospheric coupling of hydromagnetic waves-Initial results, Geophys. Res. Lett., 12, 287-290, 1985. 
Allan, W., S. P. White, and E. M. Poulter, Impulse-excited hydromagnetic cavity and field-line resonances in the magnetosphere, Planet. Space Sci., 34, 371-385, 1986.

Baumjohann, W. and K.-H. Glassmeier, The transient response mechanism and Pi2 pulsations at substorm onsetReview and outlook, Planet. Space Sci., 32, 1361-1370, 1984.

Garbow, B. S., G. Giunta, J. N. Lyness, and A. Murli, Software for an implementation of Weeks' method for the inverse Laplace transform problem, ACM Trans. Math. Software, 14, 163-170, 1988.

Itonaga, M., Parameter estimation of exponentially damped sinusoids using a modified Pisarenko method, Technol. Rep. Kyushu Univ., 63, 567-571, 1990 (in Japanese with English abstract).

Itonaga, M., Control of smoothness in nonlinear filtering of geomagnetic data by a one-pass method with a piecewise cubic polynomial, Geophys. J. Int., 1997 (in press).

Itonaga, M. and T.-I. Kitamura, Smoothing of geomagnetic data using a piecewise cubic polynomial, Geophys. J. Int., 116, 655-662, 1994.

Itonaga, M., A. Yoshikawa, and K. Yumoto, One-dimensional transient response of the inner magnetosphere at the magnetic equator, 1. Transfer function and poles, J. Geomag. Geoelectr., 49, this issue, 21-48, 1997.

Lyness, J. N. and G. Giunta, A modification of the Weeks method for numerical inversion of the Laplace transform, Math. Comput., 47, 313-322, 1986.

Rissanen, J., Modeling by shotest data description, Automatica, 14, 465-471, 1978.

Takahashi, K., S. Ohtani, and K. Yumoto, AMPTE CCE observations of Pi 2 pulsations in the inner magnetosphere, Geophys. Res. Lett., 19, 1447-1450, 1992.

Takahashi, K., S. Ohtani, and B. J. Anderson, Statistical analysis of Pi 2 pulsations observed by the AMPTE CCE spacecraft in the inner magnetosphere, J. Geophys. Res., 100, 21,929-21,941, 1995.

Weeks, W. T., Numerical inversion of Laplace transforms using Laguerre functions, J. Assoc. Comput. Mach., 13, $419-429,1966$ 